\title{
Higher-Order Phase-Space Reconstruction for Detection of Epileptic Electroencephalogram
}

\author{
Nazia Parveen ${ }^{a}$, and S.H. Saeed ${ }^{b}$ \\ a,b \\ Integral University, Lucknow, India
}

\begin{abstract}
Article History: Received: 11 January 2021; Accepted: 27 February 2021; Published online: 5 April 2021
Abstract: In this paper, the authors propose a new technique for the classification of seizures, non-seizures, and seizure-free EEG signals based on non-linear trajectories of EEG signals. The EEG signals are decomposed using the EMD technique to obtain intrinsic mode functions (IMFs). The phase space of these IMFs is then reconstructed using a novel technique of higherorder dimensions (3D, 4D, 5D, 6D, 7D, 8D, 9D, and 10D). The existing techniques of seizure detection have deployed 2D \& 3D phase-space reconstruction only. The Euclidean distance of all higher-order PSR is used as a feature to classify seizures, non-seizures, and seizure-free EEG signals. The performance of the proposed method is analyzed on the Bonn University database in which 7D reconstructed phase space classification accuracy of $99.9 \%$ has been achieved both using Random Forest classifier and $\mathrm{J} 48$ decision tree.
\end{abstract}

Keywords: electroencephalogram (EEG), phase space, Euclidean distance, inter-quartile range

\section{Introduction}

Epilepsy is an acute nervous disorder occurring due to episodes of neuronal discharges within the brain. Around $0.8 \%$ of the world population is suffering from epilepsy. A very high percentage of epileptic patients belong to middle-income countries and therefore, unable to receive the required medication [1]. The uncertainty and random occurrence associated with seizures are the main cause of causality. This uncertainty can be reduced by devising systems for automated detection \& prediction of epileptic seizures. The symptom of epilepsy is a subject of debate for various surgeons. Epilepsy is a crucial problem and posing a burden in various countries across the world including South Africa, the US, Pakistan, etc. In Pakistan, this rate is $0.99 \%$ [2].

The analysis of EEG signals involves feature extraction and classification using time-domain, frequencydomain \& time-frequency domain methods. The dynamics of EEG signals can be captured more accurately by non-linear signals. In the literature, there exist various non-linear techniques [3-8] mostly based on phase-space to detect seizures. The most common and highly explored methods based on non-linear signal analysis include principal component analysis, Lyapunov exponent, etc. Lyapunov exponent has shown promising results in its ability to characterize the dynamics of the pre-ictal state by measuring the divergence in trajectories of the dynamical system. The first-order derivative along with phase-space reconstruction using Euclidean distance had been used in [9]. In [10,11] the classification of ictal and seizure-free EEG signals using a second-order difference plot (SODP) has been used. Recently, permutation entropy [12] has been computed to distinguish pre-ictal and normal EEG signals. In [13] the convolutional neural-network-based approach is used to detect Nocturnal Frontal Lobe Epilepsy (NFLE). The challenges in accurate seizure detection and prediction are enormous. In [14], seizure onset has been identified using synchrony measures from different epileptogenic zones.

The brain is the most complex system in the human body that has adaptive, nonlinear, and dynamic characteristics. It can be considered as a self-organizing spatially embedded network at different temporal and spatial scales. It is difficult to analyze such a system due to the formation of complex dynamic structures. Hence space reconstruction technique or embedding time series is used to represent the state space of a dynamic system [7]. The latest technique [15] on phase space elliptic density has used a meta-heuristic optimization method for the detection of epileptic EEG signals. The time-scale decomposition, discrete wavelet transform (DWT),phasespace reconstruction, and neural networks have been utilized to discriminate normal, ictal, and inter-ictal EEG signals [16]. The most common procedure adopted for nonlinear dynamical analysis is the time-delay approach. Hence, in this paper phase space is reconstructed for seizure and non-seizure EEG signals using 2D, 3D,4D,5D ,6D,7D,8D,9D,10D phase space plots.

\section{Research Method}

\subsection{EEG Database}

The study has been performed on the Bonn University EEG database publicly available and described in [17]. The data has been recorded using a sampling frequency of $173.61 \mathrm{~Hz}$ for $23.6 \mathrm{sec}$ using 100 single-channel EEG electrodes. It has five subsets: Z, O, N, and F for non-seizures and S for seizures, each consisting of 100 singlechannel EEG signals.

\subsection{Phase Space Representation}


According to earlier studies, the phase space technique can be used to extract features for the classification of healthy subjects and epileptic patients. The phase space reconstruction method was proposed by Takens et al. [18]. The auto-regressive modeling of the phase space has been utilized in literature [19] for the classification of 3-class EEG-based motor imagery. In a nonlinear system, the time-series signal is converted to a phase space series signal using the phase space reconstruction method. The nonlinear systems tend to gravitate towards specific regions in phase space known as attractors. Attractors are usually represented using phase space plots of the nonlinear system. The dynamics of a nonlinear EEG signal can be visualized through these phase space trajectories which are computer-generated plots of EEG. The geometrical phase space EEG patterns are plotted when a point traces a trajectory in space. These patterns describe the state of the system as it evolves. The degrees of chaos or complexity can be quantified in terms of dimensionality [20]. N-dimensional phase space consists of d-independent variables. For the phase space reconstruction, the time-delay method is frequently used [18] viz. for the 1-D time series, other dimensions may be obtained by delayed counterparts of the observed variable. Let $\mathrm{F}[\mathrm{n}]$ is the signal to be analyzed which can be represented as a vector in space as:

$$
\mathrm{F}[\mathrm{n}]=\{\mathrm{f}[\mathrm{n}], \mathrm{f}[\mathrm{n}+\mathrm{t}], \ldots . \mathrm{f}[\mathrm{n}+(\mathrm{d}-1) \mathrm{t}\}
$$

where $\mathrm{n}=1,2,3 \ldots \mathrm{N}-(\mathrm{d}-1) \mathrm{t}$, $\mathrm{d}$ is called the embedding dimension, and $\mathrm{t}$ is the lag factor.

For the time series matched as $\{\mathrm{f} 1, \mathrm{f} 2, \ldots, \mathrm{fN}\}$ there are vectors described as:

$\mathrm{Yi}=[\mathrm{fi} \mathrm{fi}+\tau \ldots \mathrm{fi}+(\mathrm{d}-1) \tau]$,

where $\tau=\mathrm{i}$ Ts is the reconstruction of delay or lag, $\mathrm{n}=1,2, \ldots, \mathrm{N}-(\mathrm{d}-1) \tau$ and $\mathrm{d}$ is the embedding dimension.

In this method choosing proper values of time -delay and embedding dimension is very important. If timedelay $(t)$ is chosen very small, then coordinates of vector $X[n]$ are nearly equal and the reconstructed phase space vectors are too close to obtain dynamic information of the signal. On the other hand, for a too-large value of timedelay, the reconstructed phase space vectors are too far apart. Hence original phase reconstruction is largely dependent on the choosing correct value of time-delay. Also, it is important to choose a sufficient embedding dimension in phase space to represent the dynamics of the system. The criterion for select the embedding dimension is based on properties of the "non-cutting" trajectory and reversibility of the reconstructed attractors. In this embedded dimension $\mathrm{d}$ is chosen 2 and 3 for visualization simplicity and time-lag $\square=1$ was used for the construction of an EEG attractor.

The $2 \mathrm{D}$ phase space plot can be obtained between two variables $(\mathrm{xn}, \mathrm{xn}+1)$ while a 3D phase space plot is plotted between $x n$ and its delayed versions by one \& two represented as $x n+1 \& x n+2$ respectively [21]. A new vector is created in space by time-delayed values of scalar measurements.

\subsection{Feature extraction for EEG Signals}

\subsubsection{Area computation of elliptical pattern for 2D PSR}

The IMFs of EEG signal obtained after decomposition is oscillatory. Hence the PSR of IMFs is also elliptical. 2D PSR is a plot between the two vectors $\mathrm{Vk} \& \mathrm{Vk}+1[22$ ]. The 2D PSR plot of the first four IMFs computed for the $95 \%$ confidence area has been shown in Fig.1. It is depicted from the figure that the area of elliptical patterns differs for each signal. The larger area for seizure EEG helps in discriminating it from normal EEG.

\subsubsection{Computation of Euclidean Distance \& IQR for higher dimension PSR (3D-10D)}

3D PSR plot is used to represent the dynamics of the system. It is plotted between three vectors delayed by one unit each i.e. rk,rk+1,rk+2. The distance between origin and the point (rk,rk+1,rk+2) is defined as Euclidean distance and computed as

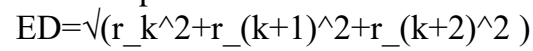

Fig.2. shows the Euclidean distance plot for 3D PSR. It can be seen that the length of Euclidean distance is different for each class of EEG signals.

IQR of Euclidean distance

IQR, based on Euclidean distance is the measure of the variability of data. It is the difference between the first $\&$ third quartiles based on dividing the dataset into quartiles. Mathematically, IQR can be expressed as:

$\mathrm{IQR}=\mathrm{Q} 3-\mathrm{Q} 1$

\section{Results \& Discussion}

In this approach, the five sets of the Bonn University EEG database are used. In the first stage, empirical mode decomposition of EEG signals is performed to extract intrinsic mode functions. The first four IMFs are used for feature extraction in phase space. The 2D phase space is an elliptical pattern and the area of the ellipse is computed for all EEG signals. For higher-order phase space, Euclidean distance is computed for all EEG signals. The Euclidean distance extracted for all EEG signals is given as input to JRIP, J48 \& Random Forest classifier. The comparative performance of classifiers has been done in Table 1 for 3D PSR. 
(a)

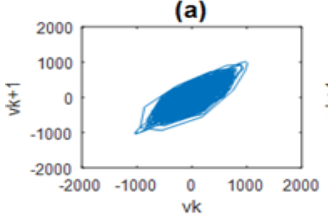

(c)

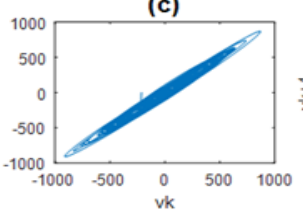

(b)

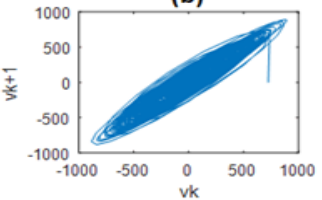

(d)

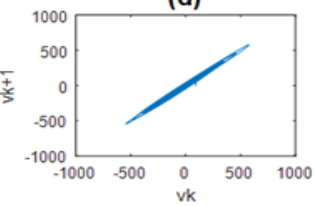

(i)

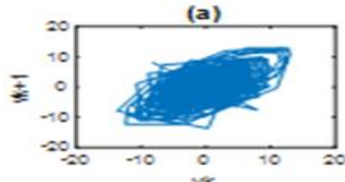

(c)
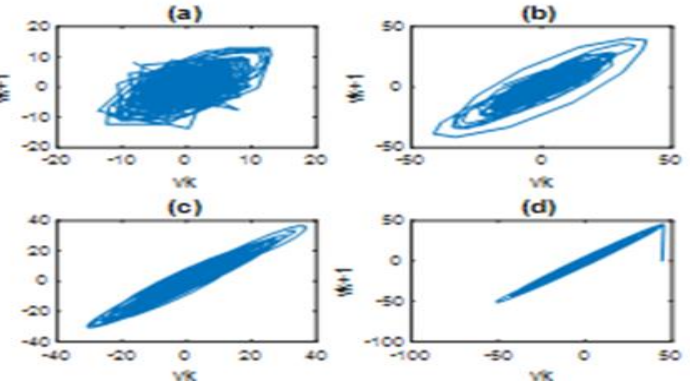

(d)

(ii)

Fig.1. 2D PSR (i) seizure EEG (ii) seizure-free EEG

Fig. 1 shows that the intrinsic mode functions of 2D PSR exhibit elliptical patterns in which a 95\% confidence area can be used as a feature for the classification of the epileptic seizure and seizure-free EEG signals.

(a)

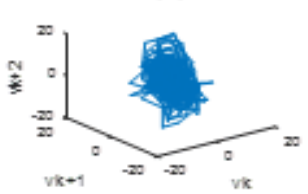

(c) (b)

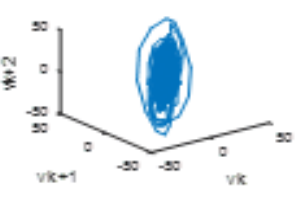

(d)

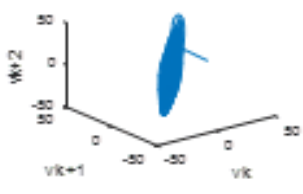

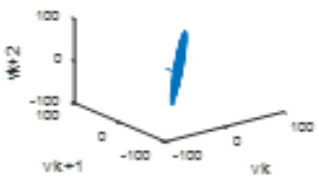

(i)

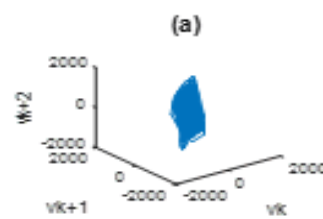

(c)

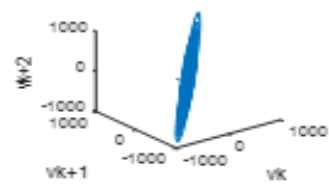

(ii)

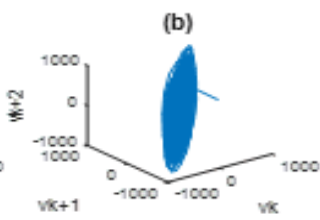

(d)

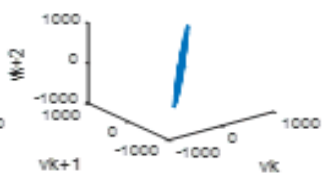

Fig.2. 3D PSR (i) seizure EEG (ii) seizure-free EEG

Fig. 2 shows elliptical balls obtained for 3D PSR discriminate seizure and seizure-free EEG signals based on interquartile range (IQR) of Euclidean distance (ED).

Table 1 shows performance measures for 3D-10D PSR. It can be observed that the Random Forest classifier gives maximum classification accuracy for 3D PSR while for 4D PSR 99.9\% classification accuracy has been achieved using an RF classifier. The graphical comparison of performance has been done in Fig 3 .

\subsection{Performance Evaluation}

Accuracy, sensitivity, and specificity are the performance parameters of the classifier and have been defined as [23]

Accuracy $(\%)=(\mathrm{TP}+\mathrm{TN}) /(\mathrm{TP}+\mathrm{TN}+\mathrm{FP}+\mathrm{FN}) \times 100$

Sensitivity $(\%)=\mathrm{TP} /(\mathrm{TP}+\mathrm{FN}) \times 100$

Specificity $(\%)=\mathrm{TN} /(\mathrm{TN}+\mathrm{FP}) \times 100$

where TP $\rightarrow$ true positive, the number of epochs which are marked correctly as seizure by both neurologist and algorithm.

$\mathrm{TN} \rightarrow$ true negative, the number of epochs that are marked as non-seizure by both neurologist and algorithm.

$\mathrm{FN} \rightarrow$ false negative, the number of seizure epochs that are misclassified by the algorithm, i.e. recognized as non-seizure but actually, they are seizures.

$\mathrm{FP} \rightarrow$ false positive, the number of non-seizures epochs which are misclassified by the algorithm, i.e. recognized as seizure but actually, they are non-seizures.

Table 1. Performance Comparison of Classifiers (a) 3D PSR(b) 4D PSR(c) 5D PSR(d) 6D PSR (e) 7D PSR (f) 8D PSR (g) 9D PSR (h) 10D PSR

\begin{tabular}{|l|l|l|l|l|l|l|l|l|l|}
\hline \multirow{2}{*}{ 3D PSR } & \multicolumn{3}{|c|}{ JRIP } & \multicolumn{3}{c|}{ RF } & \multicolumn{3}{c|}{ J48 } \\
\cline { 2 - 10 } & $\begin{array}{l}\text { Acc } \\
\%\end{array}$ & $\begin{array}{l}\text { Sen } \\
\%\end{array}$ & $\begin{array}{l}\text { Acc } \\
\%\end{array}$ & $\begin{array}{l}\text { Sen } \\
\%\end{array}$ & $\begin{array}{l}\text { Spe } \\
\%\end{array}$ & $\begin{array}{l}\text { Acc } \\
\%\end{array}$ & $\begin{array}{l}\text { Sen } \\
\%\end{array}$ & $\begin{array}{l}\text { Spe } \\
\%\end{array}$ \\
\hline Seizure & 99.7 & 99.14 & 99.9 & 99.8 & 99.5 & 99.9 & 99.7 & 99.3 & 99.8 \\
\hline $\begin{array}{l}\text { Seizure- } \\
\text { Free }\end{array}$ & 91.9 & 91.3 & 92.2 & 92.8 & 91.8 & 93.2 & 91.9 & 91.8 & 91.9 \\
\hline Normal & 91.6 & 84.4 & 95.2 & 92.6 & 86.5 & 95.6 & 91.7 & 83.8 & 95.7 \\
\hline
\end{tabular}


(a)

\begin{tabular}{|l|l|l|l|l|l|l|l|l|l|}
\hline \multirow{2}{*}{ 4D PSR } & \multicolumn{3}{|c|}{ JRIP } & \multicolumn{3}{c|}{ RF } & \multicolumn{3}{c|}{ J48 } \\
\cline { 2 - 10 } & $\begin{array}{l}\text { Acc } \\
\%\end{array}$ & $\begin{array}{l}\text { Sen } \\
\%\end{array}$ & $\begin{array}{l}\text { Spe } \\
\%\end{array}$ & $\begin{array}{l}\text { Acc } \\
\%\end{array}$ & $\begin{array}{l}\text { Sen } \\
\%\end{array}$ & $\begin{array}{l}\text { Spe } \\
\%\end{array}$ & $\begin{array}{l}\text { Acc } \\
\%\end{array}$ & $\begin{array}{l}\text { Sen } \\
\%\end{array}$ & $\begin{array}{l}\text { Spe } \\
\%\end{array}$ \\
\hline Seizure & 99.6 & 99.2 & 99.8 & 99.9 & 99.8 & 99.9 & 99.8 & 99.6 & 99.9 \\
\hline $\begin{array}{l}\text { Seizure- } \\
\text { Free }\end{array}$ & 90.2 & 88.5 & 90.9 & 91.3 & 90.0 & 92.0 & 90.1 & 88.9 & 90.7 \\
\hline Normal & 89.9 & 81.8 & 93.9 & 91.3 & 84.1 & 94.9 & 90.0 & 81.4 & 94.3 \\
\hline
\end{tabular}

(b)

\begin{tabular}{|l|l|l|l|l|l|l|l|l|l|}
\hline \multirow{2}{*}{ 5D PSR } & \multicolumn{3}{|l|}{ JRIP } & \multicolumn{2}{l|}{ RF } & \multicolumn{2}{l|}{ J48 } \\
\cline { 2 - 10 } & $\begin{array}{l}\text { Acc } \\
\%\end{array}$ & $\begin{array}{l}\text { Sen } \\
\%\end{array}$ & $\begin{array}{l}\text { Spe } \\
\%\end{array}$ & $\begin{array}{l}\text { Acc } \\
\%\end{array}$ & $\begin{array}{l}\text { Sen } \\
\%\end{array}$ & $\begin{array}{l}\text { Spe } \\
\%\end{array}$ & $\begin{array}{l}\text { Acc } \\
\%\end{array}$ & $\begin{array}{l}\text { Sen } \\
\%\end{array}$ & $\begin{array}{l}\text { Spe } \\
\%\end{array}$ \\
\hline Seizure & 99.7 & 99.5 & 99.7 & 99.8 & 99.6 & 99.9 & 99.7 & 99.4 & 99.9 \\
\hline $\begin{array}{l}\text { Seizure- } \\
\text { Free }\end{array}$ & 92.5 & 91.9 & 92.9 & 94.0 & 93.5 & 94.3 & 92.6 & 91.0 & 93.4 \\
\hline Normal & 92.3 & 85.5 & 95.8 & 93.9 & 88.7 & 96.5 & 92.5 & 86.9 & 95.3 \\
\hline
\end{tabular}

(c)

\begin{tabular}{|l|l|l|l|l|l|l|l|l|l|}
\hline \multirow{2}{*}{ 6D PSR } & \multicolumn{3}{|c|}{ JRIP } & \multicolumn{3}{c|}{ RF } & \multicolumn{3}{c|}{ J48 } \\
\cline { 2 - 10 } & $\begin{array}{l}\text { Acc } \\
\%\end{array}$ & $\begin{array}{l}\text { Sen } \\
\%\end{array}$ & $\begin{array}{l}\text { Spe } \\
\%\end{array}$ & $\begin{array}{l}\text { Acc } \\
\%\end{array}$ & $\begin{array}{l}\text { Sen } \\
\%\end{array}$ & $\begin{array}{l}\text { Spe } \\
\%\end{array}$ & $\begin{array}{l}\text { Acc } \\
\%\end{array}$ & $\begin{array}{l}\text { Sen } \\
\%\end{array}$ & $\begin{array}{l}\text { Spe } \\
\%\end{array}$ \\
\hline Seizure & 99.6 & 99.2 & 99.7 & 99.9 & 99.6 & 100 & 99.8 & 99.6 & 99.9 \\
\hline $\begin{array}{l}\text { Seizure- } \\
\text { Free }\end{array}$ & 96.7 & 91.8 & 99.3 & 94.7 & 94.2 & 94.9 & 93.1 & 91.4 & 93.9 \\
\hline Normal & 92.7 & 86.7 & 95.6 & 94.6 & 89.9 & 96.9 & 92.9 & 87.9 & 95.5 \\
\hline
\end{tabular}

(d)

\begin{tabular}{|l|l|l|l|l|l|l|l|l|l|}
\hline \multirow{2}{*}{ 7D PSR } & \multicolumn{3}{|c|}{ JRIP } & \multicolumn{3}{c|}{ RF } & \multicolumn{3}{c|}{ J48 } \\
\cline { 2 - 10 } & $\begin{array}{l}\text { Acc } \\
\%\end{array}$ & $\begin{array}{l}\text { Sen } \\
\%\end{array}$ & $\begin{array}{l}\text { Spe } \\
\%\end{array}$ & $\begin{array}{l}\text { Acc } \\
\%\end{array}$ & $\begin{array}{l}\text { Sen } \\
\%\end{array}$ & $\begin{array}{l}\text { Spe } \\
\%\end{array}$ & $\begin{array}{l}\text { Acc } \\
\%\end{array}$ & $\begin{array}{l}\text { Sen } \\
\%\end{array}$ & $\begin{array}{l}\text { Spe } \\
\%\end{array}$ \\
\hline Seizure & 99.0 & 99.6 & 99.9 & 99.9 & 99.8 & 100 & 99.9 & 99.7 & 99.9 \\
\hline $\begin{array}{l}\text { Seizure- } \\
\text { Free }\end{array}$ & 98.8 & 97.9 & 99.2 & 99.2 & 98.4 & 99.5 & 98.6 & 97.4 & 99.2 \\
\hline Normal & 98.6 & 98.3 & 98.8 & 99.1 & 99.0 & 99.1 & 98.5 & 98.5 & 98.6 \\
\hline
\end{tabular}

(e)

\begin{tabular}{|l|l|l|l|l|l|l|l|l|l|}
\hline \multirow{2}{*}{ 8D PSR } & \multicolumn{3}{|c|}{ JRIP } & \multicolumn{3}{c|}{ RF } & \multicolumn{3}{c|}{ J48 } \\
\cline { 2 - 10 } & $\begin{array}{l}\text { Acc } \\
\%\end{array}$ & $\begin{array}{l}\text { Sen } \\
\%\end{array}$ & $\begin{array}{l}\text { Spe } \\
\%\end{array}$ & $\begin{array}{l}\text { Acc } \\
\%\end{array}$ & $\begin{array}{l}\text { Sen } \\
\%\end{array}$ & $\begin{array}{l}\text { Spe } \\
\%\end{array}$ & $\begin{array}{l}\text { Acc } \\
\%\end{array}$ & $\begin{array}{l}\text { Sen } \\
\%\end{array}$ & $\begin{array}{l}\text { Spe } \\
\%\end{array}$ \\
\hline Seizure & 99.6 & 99.4 & 99.7 & 99.8 & 99.6 & 99.9 & 99.8 & 99.5 & 99.9 \\
\hline $\begin{array}{l}\text { Seizure- } \\
\text { Free }\end{array}$ & 94.6 & 94.1 & 94.9 & 96.4 & 96.4 & 96.4 & 95.0 & 93.8 & 95.5 \\
\hline Normal & 94.3 & 89.5 & 96.8 & 96.4 & 92.9 & 98.0 & 94.8 & 91.1 & 96.6 \\
\hline
\end{tabular}

(f)

\begin{tabular}{|l|l|l|l|l|l|l|l|l|l|}
\hline \multirow{2}{*}{ 9D PSR } & \multicolumn{3}{|c|}{ JRIP } & \multicolumn{3}{c|}{ RF } & \multicolumn{3}{c|}{ J48 } \\
\cline { 2 - 10 } & $\begin{array}{l}\text { Acc } \\
\%\end{array}$ & $\begin{array}{l}\text { Sen } \\
\%\end{array}$ & $\begin{array}{l}\text { Spe } \\
\%\end{array}$ & $\begin{array}{l}\text { Acc } \\
\%\end{array}$ & $\begin{array}{l}\text { Sen } \\
\%\end{array}$ & $\begin{array}{l}\text { Spe } \\
\%\end{array}$ & $\begin{array}{l}\text { Acc } \\
\%\end{array}$ & $\begin{array}{l}\text { Sen } \\
\%\end{array}$ & $\begin{array}{l}\text { Spe } \\
\%\end{array}$ \\
\hline Seizure & 99.7 & 99.6 & 99.8 & 99.9 & 99.7 & 100 & 99.8 & 99.5 & 99.9 \\
\hline $\begin{array}{l}\text { Seizure- } \\
\text { Free }\end{array}$ & 94.1 & 94.0 & 94.1 & 96.1 & 95.9 & 96.3 & 94.7 & 93.9 & 95.1 \\
\hline Normal & 94.3 & 89.0 & 96.9 & 96.0 & 92.5 & 97.8 & 94.5 & 90.0 & 96.7 \\
\hline
\end{tabular}


(g)

\begin{tabular}{|l|l|l|l|l|l|l|l|l|l|}
\hline \multirow{2}{*}{$\begin{array}{l}\text { 10D } \\
\text { PSR }\end{array}$} & $\begin{array}{l}\text { Acc } \\
\%\end{array}$ & $\begin{array}{l}\text { Sen } \\
\%\end{array}$ & $\begin{array}{l}\text { Spe } \\
\%\end{array}$ & $\begin{array}{l}\text { Acc } \\
\%\end{array}$ & $\begin{array}{l}\text { Sen } \\
\%\end{array}$ & $\begin{array}{l}\text { Spe } \\
\%\end{array}$ & $\begin{array}{l}\text { Acc } \\
\%\end{array}$ & $\begin{array}{l}\text { Sen } \\
\%\end{array}$ & $\begin{array}{l}\text { Spe } \\
\%\end{array}$ \\
\hline Seizure & 99.7 & 99.4 & 99.8 & 99.9 & 99.8 & 100 & 99.8 & 99.7 & 99.9 \\
\hline $\begin{array}{l}\text { Seizure- } \\
\text { Free }\end{array}$ & 94.4 & 93.9 & 94.6 & 96.6 & 96.5 & 96.7 & 94.9 & 94.3 & 95.2 \\
\hline Normal & 94.2 & 89.1 & 96.8 & 96.6 & 93.4 & 98.1 & 94.8 & 90.4 & 97.0 \\
\hline
\end{tabular}

(h)

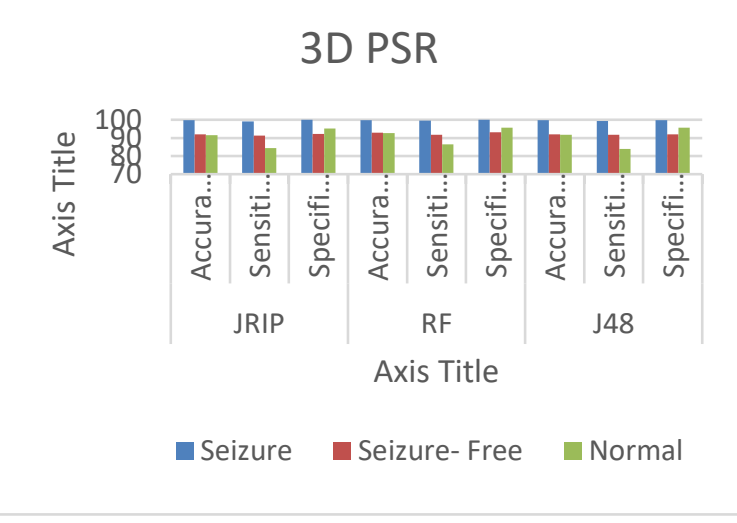

(a)

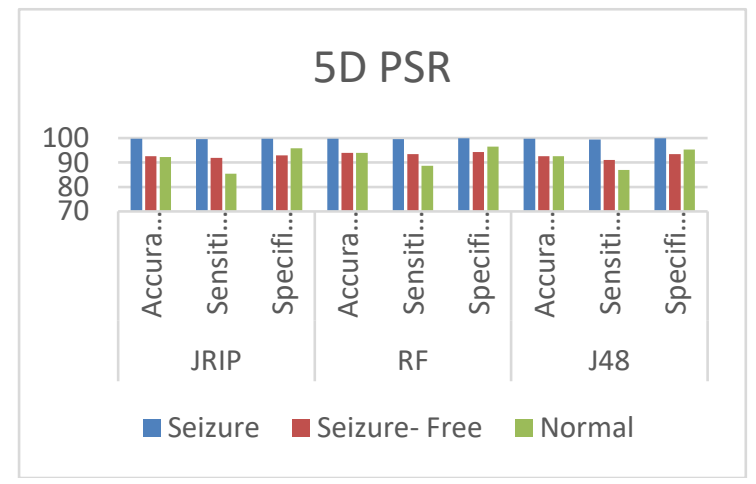

(c)

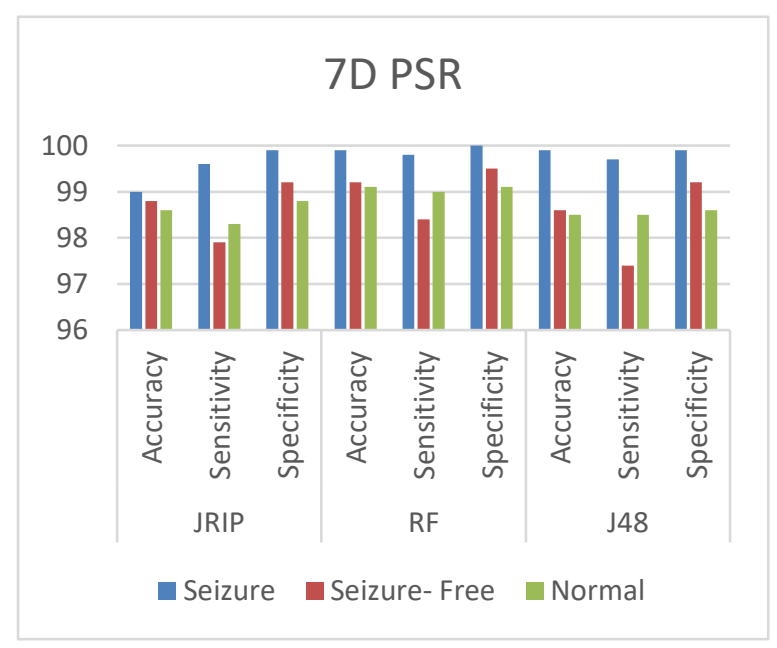

(e)

\section{D PSR}

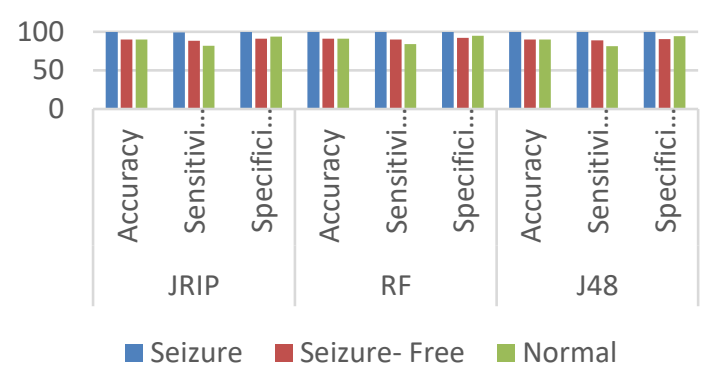

(b)

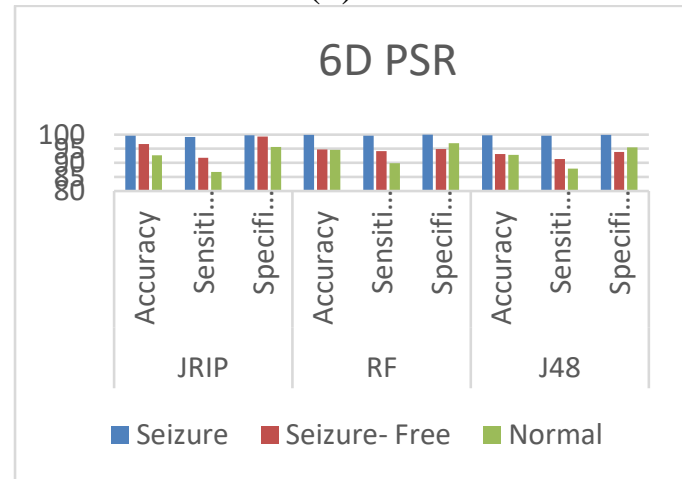

(d)

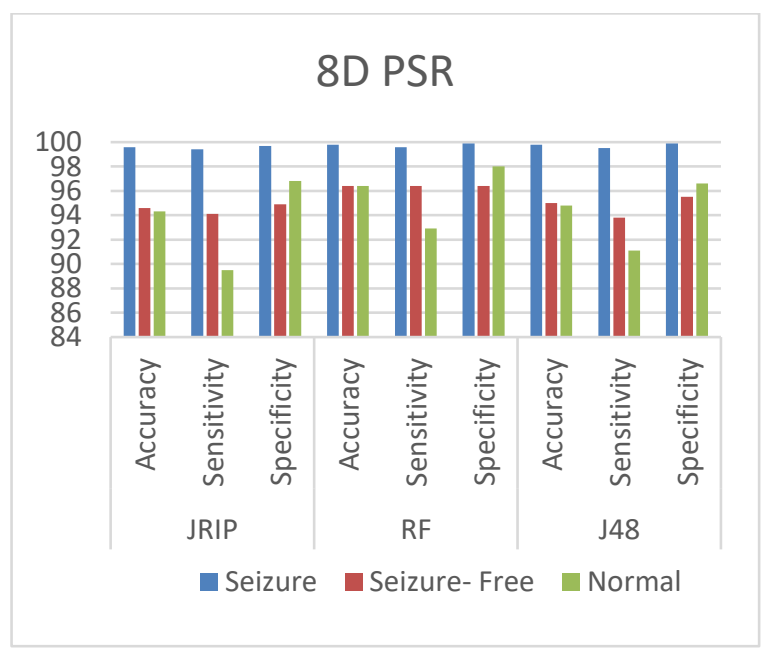

(f) 


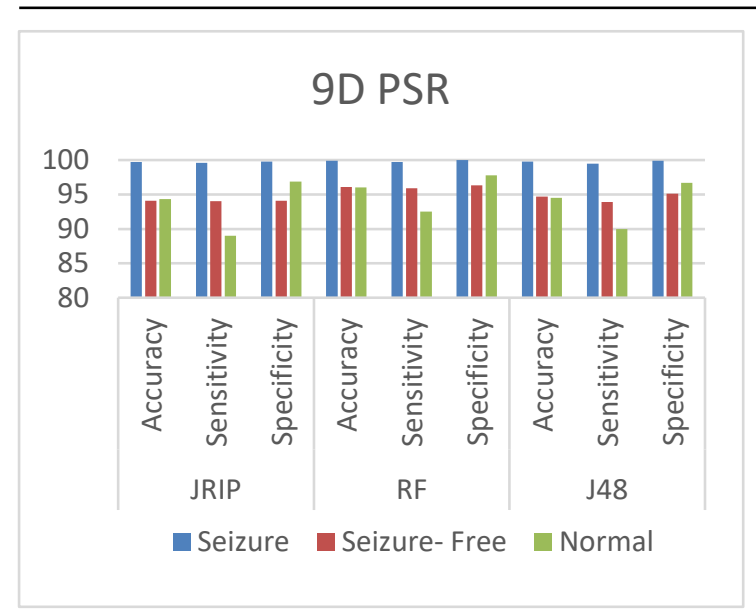

$(\mathrm{g})$

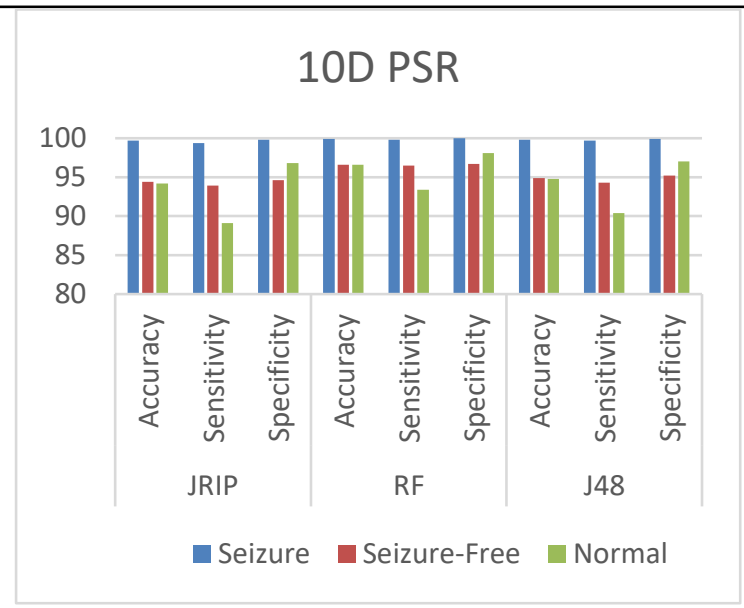

(h)

Fig.3. Performance Analysis of higher order PSR (a) 3D PSR(b) 4D PSR(c) 5D PSR(d) 6D PSR (e) 7D PSR (f) 8D PSR (g) 9D PSR (h) 10D PSR

Table 2. Comparison of the proposed method with the existing methods studied on the same dataset

\begin{tabular}{|l|l|l|l|}
\hline Author & Method & Dataset & $\begin{array}{l}\text { Accuracy } \\
(\%)\end{array}$ \\
\hline Salim Rukhsar (2018) [16] & $\begin{array}{l}\text { First-order difference-phase space } \\
\text { reconstruction, decision tree-ECOC }\end{array}$ & A, B, C, D, E & 100 \\
\hline Pachori et al. (2015) [9] & $\begin{array}{l}\text { EMD based Euclidean distance and 95\% of } \\
\text { confidence area, support vector machine }\end{array}$ & C,D \& E & 98.67 \\
\hline S. H. Lee et al.(2014) [7] & The Euclidean distance of PSR with NEWFM & A \& E & 98.17 \\
\hline R.B. Pachori et al. (2014) [3] & $\begin{array}{l}\text { 95\% of confidence area measure of SODP of } \\
\text { IMFs with ANN }\end{array}$ & C,D \& E & 95.75 \\
\hline Proposed Methodology & $\begin{array}{l}\text { For 2D PSR 95\% confidence area of the } \\
\text { ellipse is computed while for higher-order PSR } \\
\text { IQR of ED is computed }\end{array}$ & A, B, C, D, E & 99.9 \\
\hline
\end{tabular}

\section{Conclusion}

Due to the widely accepted fact about the chaotic nature of the brain, its non-linear dynamics have been studied using phase-space representation of higher dimensions. This method intends to improve the classification accuracy of seizure, seizure-free, and non-seizure EEG signals. The novelty of this approach is the use of higherorder PSRs for the detection of epileptic seizures. The first four IMFS are obtained using the EMD technique and 2D PSR plot elliptical area is computed. However, for higher-order PSR, IQR of Euclidean distance is computed. For classification JRIP, the J48 decision tree \& Random Forest classifier are used to compare the accuracy, sensitivity, and specificity of different EEG signals. It has been found that the Random Forest Classifier and J48 decision tree perform better as compared to JRIP (Table 1). The accuracy achieved by using 10 fold crossvalidation in 4D,5D 6D, 7D, 8D, 9D \& 10D phase space reconstruction is higher as compared to the existing methods based on 2D \& 3D PSR.

\section{References}

1. World Health Organization: WHO. “Epilepsy.”WHO, 20 June 2019, www.who.int/news-room/factsheets/detail/epilepsy.

2. Khatri, I A et al. "Epidemiology of epilepsy in Pakistan: a review of the literature." JPMA. The Journal of the Pakistan Medical Association vol. 53,12 (2003): 594-7.

3. Zabihi, Morteza, et al. "Analysis of High Dimensional Phase Space via Poincaré Section for PatientSpecific Seizure Detection." IEEE Transactions on Neural Systems and Rehabilitation Engineering, vol. 24, no. 3, 2016, pp. 386-98. Crossref, doi:10.1109/tnsre.2015.2505238.

4. Wang, Lina, et al. "Automatic Epileptic Seizure Detection in EEG Signals Using Multi-Domain Feature Extraction and Nonlinear Analysis." Entropy, vol. 19, no. 6, 2017, p. 222. Crossref, doi:10.3390/e19060222.

5. Ilakiyaselvan, N., et al. "Deep Learning Approach to Detect Seizure Using Reconstructed Phase Space Images." The Journal of Biomedical Research, vol. 34, no. 3, 2020, p. 240. Crossref, doi:10.7555/jbr.34.20190043. 
6. Kennel, Matthew B., et al. "Determining Embedding Dimension for Phase-Space Reconstruction Using a Geometrical Construction.” Physical Review A, vol. 45, no. 6, 1992, pp. 3403-11. Crossref, doi:10.1103/physreva.45.3403.

7. Kumar, S. T. Sadish, and N. Kasthuri. "EEG Seizure Classification Based on Exploiting Phase Space Reconstruction and Extreme Learning." Cluster Computing, vol. 22, no. S5, 2017, pp. 11477-87. Crossref, doi:10.1007/s10586-017-1409-z.

8. Lee, Sang-Hong, et al. "Classification of Normal and Epileptic Seizure EEG Signals Using Wavelet Transform, Phase-Space Reconstruction, and Euclidean Distance." Computer Methods and Programs in Biomedicine, vol. 116, no. 1, 2014, pp. 10-25. Crossref, doi:10.1016/j.cmpb.2014.04.012.

9. Rukhsar, Salim, et al. "Detection of Epileptic Seizure in EEG Signals Using Phase Space Reconstruction and Euclidean Distance of First-Order Derivative.” 2017 International Conference on Inventive Computing and Informatics (ICICI), 2017. Crossref, doi:10.1109/icici.2017.8365251.

10. Pachori, R. B.; Patidar, S. Epileptic seizure classification in EEG signals using a second-order difference plot of intrinsic mode functions. Comput. Methods Programs Biomed. 2014, 113 (2), 494502. https://doi.org/10.1016/j.cmpb.2013.11.014.

11. Rukhsar, Salim. "Discrimination of Multi-Class EEG Signal in Phase Space of Variability for Epileptic Seizure Detection Using Error-Correcting Output Code (ECOC).” International Journal of Information Technology, 2018. Crossref, doi:10.1007/s41870-018-0224-y.

12. Redelico, F.; Traversaro, F.; García, M.; Silva, W.; Rosso, O.; Risk, M. Classification of Normal and Pre-Ictal EEG Signals Using Permutation Entropies and a Generalized Linear Model as a Classifier. Entropy 2017, 19 (2), 72. https://doi.org/10.3390/e19020072.

13. Pisano, F.; Sias, G.; Fanni, A.; Cannas, B.; Dourado, A.; Pisano, B.; Teixeira, C. A. Convolutional Neural Network for Seizure Detection of Nocturnal Frontal Lobe Epilepsy. Complexity 2020, 2020, $1-$ 10. https://doi.org/10.1155/2020/4825767.

14. Sharma, Aarti, et al. "Epileptic Seizure Prediction and Identification of Epileptogenic Region Using EEG Signal.” 2015 International Conference on Green Computing and Internet of Things (ICGCIoT), 2015. Crossref, doi:10.1109/icgciot.2015.7380644.

15. Darjani, Nastaran, and Hesam Omranpour. "Phase Space Elliptic Density Feature for Epileptic EEG Signals Classification Using Metaheuristic Optimization Method.” Knowledge-Based Systems, vol. 205, 2020, p. 106276. Crossref, doi:10.1016/j.knosys.2020.106276.

16. Zeng, Wei, et al. "Identification of Epileptic Seizures in EEG Signals Using Time-Scale Decomposition (ITD), Discrete Wavelet Transform (DWT), Phase Space Reconstruction (PSR) and Neural Networks." Artificial Intelligence Review, vol. 53, no. 4, 2019, pp. 3059-88. Crossref, doi:10.1007/s10462-01909755-y.

17. Andrzejak, Ralph. "Indications of Nonlinear Deterministic and Finite-Dimensional Structures in Time Series of Brain Electrical Activity: Dependence on Recording Region and Brain State." Physical Review E, 20 Nov. 2001, journals.aps.org/ pre/abstract/10.1103/PhysRevE.64.061907.

18. Takens, Floris. “Detecting Strange Attractors in Turbulence.” SpringerLink, 12 Oct. 1981, link.springer.com/chapter/ 10.1007\%2FBFb0091924

19. Djemal, Ridha, et al. "Three-Class EEG-Based Motor Imagery Classification Using Phase-Space Reconstruction Technique.” Brain Sciences, vol. 6, no. 3, 2016, p. 36. Crossref, doi:10.3390/brainsci6030036.

20. Lam, Lui. Introduction to Nonlinear Physics. Springer, 2003.

21. Sharma, Rajeev, and Ram Bilas Pachori. "Classification of Epileptic Seizures in EEG Signals Based on Phase Space Representation of Intrinsic Mode Functions.” Expert Systems with Applications, vol. 42, no. 3, 2015, pp. 1106-17. Crossref, doi:10.1016/j.eswa.2014.08.030.

22. Krishnaprasanna, R., and V. Vijaya Baskar. "Focal and Non-Focal EEG Signal Classification by Computing Area of 2D-PSR Obtained for IMF. ” Journal of ICT Standardization, vol. 5, no. 2, 2018, pp. 171-86. Crossref, doi:10.13052/jicts2245-800x.523.

23. Ph.D., Satapathy Sandeep Kumar, et al. EEG Brain Signal Classification for Epileptic Seizure Disorder Detection. 1st ed., Academic Press, 2019. 